\title{
An Energy Management Approach for Solar Charging Stations in Smart Cities
}

\author{
${ }^{1}$ M. Tahir Guneser, ${ }^{* 1}$ Mohamed Elweddad and ${ }^{2}$ Cevat Ozarpa \\ ${ }^{*}{ }^{1}$ Karabuk University, Engineering Faculty, Electrical and Electronics Engineering Department, Karabuk, Turkiye \\ ${ }^{2}$ Karabuk University, Engineering Faculty, Mechanical Engineering Department, Karabuk, Turkiye
}

\begin{abstract}
The rapid development of intelligent transportation systems in smart cities offers great opportunities to improve the performance of energy management strategies (EMSs) for connected hybrid electric vehicles (HEV) charging systems. In this study, an electric vehicle charging station integrating solar photovoltaic (PV) panels, a Battery Energy Storage System (BESS) and a based on utility grid is designed for many scenarios. In the proposed system, the PV is the primary energy source with the battery for storing excessive energy to deliver the lowest possible electricity cost with the grid-tied to ensure power supply continuity. An efficient design of charging solar station with a control strategy is developed for the optimal power management between PV and BESS based on the utility grid. By taking the dynamic charging process of EVs battery, the power management flow in the station is formulated and validated using MATLAB.
\end{abstract}

Key words: Electrical vehicle, energy management, machine learning, renewable energy, smart city

\section{Introduction}

Hybrid Electric Vehicles (HEVs) is among the best solution to reduce environmental pollution and fossil fuel consumption. The main benefit of using HEVs is fuel savings, which this paper represents a challenge to improve the right energy management strategy (EMS) for HEVs. Furthermore, the requirements of EMS design are essential for optimal power distribution at less cost. HEVs consist of different types of energy sources using power converters, which refer to cars including internal combustion engine (ICE) with an electric motor. HEVs seem to be one of the best viable solutions for the next decades. The general aim of HEVs is reducing the fuel consumption and $\mathrm{CO}_{2}$ emissions, whilst ensuring the peak demand satisfaction by using an appropriate EMSs. Power management aims to acquire an optimal power flow in presence of complex conditions, as well as to reduce fuel consumption and gas emissions. It is commonly known that refinement in fuel consumption and reduction in gas emissions is depending on their energy management strategies (EMSs) [1]. The complex structure of multi-source energy systems comes with challenges regarding the performance of EMSs. The EMS goal is to manage the available power from the energy sources in a controlled manner [2].

Intelligent solar charging stations (CSs) that have a supervisory control on (EVs) have been developed, some of these stations integrated with vehicle-to-grid (V2G) technology. So they can provide more benefits for the utility grid and EV owners [3]. Different EMSs have been used for HEVs recently. The authors presented a number of classifications for management strategies (EMS) $[4,5]$. In general, EMSs can be divided into three categories: rule-based, domestic

*Corresponding author: Address: Faculty of Engineering, Department of Electrical and Electronics Engineering Karabuk University, 78100, Karabuk TURKIYE. E-mail address: mtguneser@karabuk.edu.tr, Phone: +905552739874 
optimization-based, and universal optimization-based [3]. Authors presented plug-in Hybrid Electric EMSs [6]. The classification of energy management, such as rule-based control strategies and optimization-based control strategies, are presented according to mathematical models and the used approach. According to The National Transportation Research Center (NTRC), the consumed energy is 27706.5 Trillion Btu for transportation sector and about 6.6 GT gas emitted, whereas EV has less impact to the environment and has a low level of noise [7]. Developing countries have faced power crises over the last decades [8]. Charging electric vehicles causes an additional load on the power grid and, result in massive load shedding. It is necessary to recharge these EVs from an alternative energy source such as PV energy resources [3]. The authors in [9] utilized Linear Programming to solve the optimization problem, whereas in Reference [10], a Dynamic Programming (DP) was used to deal with the problem. proposed a quadratic optimization problem from the costumer's point of view which aimed at minimizing the charging costs and provided valley filling.

A Mixed Discrete Programming was used to solve problems in [10] which is charging and discharging the EVs in an optimal way. In reference [11] the authors proposed scheduling of EV charging in intelligent parking to maximize the charging/discharging rate considering the batteries condition. This is done by reducing the number of used switches between charge and discharge process. The works in [12] depending on the power generated from conventional units to charge $\mathrm{EV}$. The work considering PVS as the main power source for EV charging hence is designed to fully deliver the environmental and economic features of EVs.

The main objective of this study is to propose a methodology to charge the HEVs in optimal manner from a solar energy-based station.

\section{Solar HEV Charging Station System}

Proposed grid connected energy storage system can be integrated to photovoltaic module, DC power distribution equipment, battery storage, charging station intelligent control system, charging interface and power grid interface, etc. The specific system structure is shown in Fig 1.

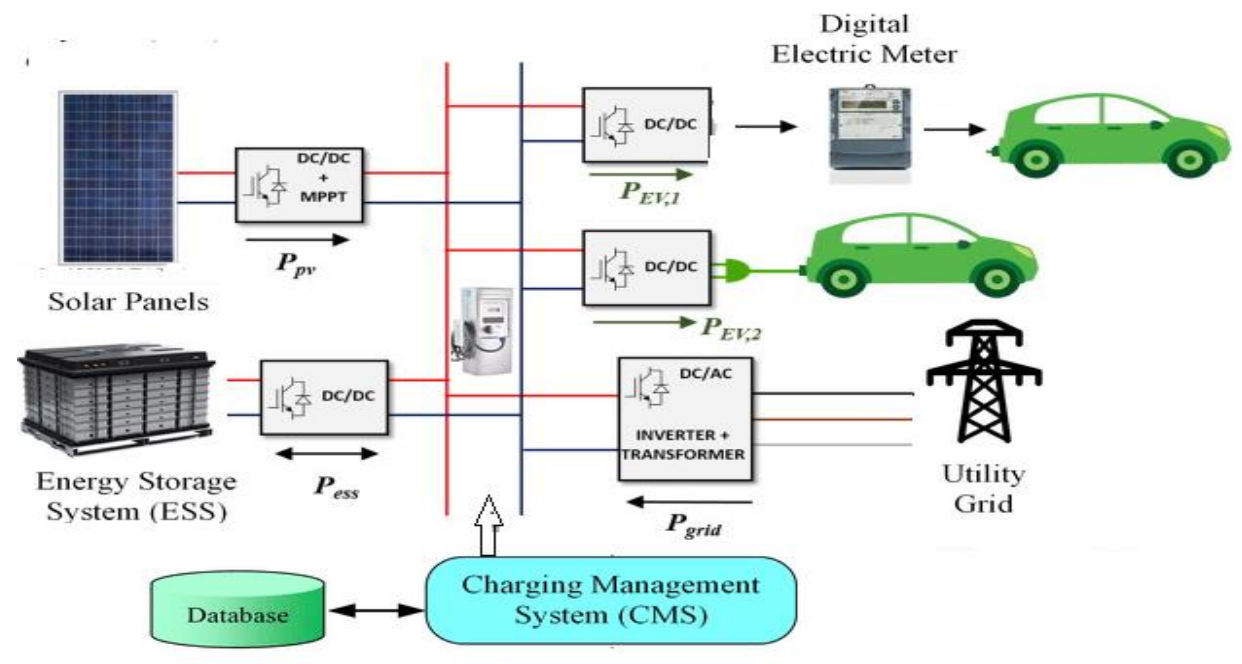

Figure 1. The system structure of a solar charging station

With a great amount of sunshine during the day, PV systems deliver DC voltage to the charging station. The intelligent control system is utilized to control the charging and discharging storage better. At night or on cloudy weather when the storage is inadequate, grid is utilized through 
the intelligent control system to supply power to the HEVs. Also, through the charging control system, the storage batteries are charged by using a bi-directional inverter, which effectively guarantees the continuous charging and stable operation.

\subsection{Design of Charging Station}

The used technique in designing charging station is quite different from conventional techniques. When a vehicle comes to the station, automatically the state of battery charge will be measured with the control mechanism. Then, the control strategy will manage its next step by defining the available energy source of solar/backup battery/grid. The priority is always PV panel, next is the backup battery and then the last option is the grid.

If shadow affects the PV output, then the backup will automatically activate. At the same time, the control technique will track whether the PV panel output increases or not. If PV panel output increases, then switch to PV otherwise not. By using this strategy, the three sources (PV, Battery, Grid) can be interfaced with resultant performance better than any other technique. Fig. 2 shows the process of charging stations.

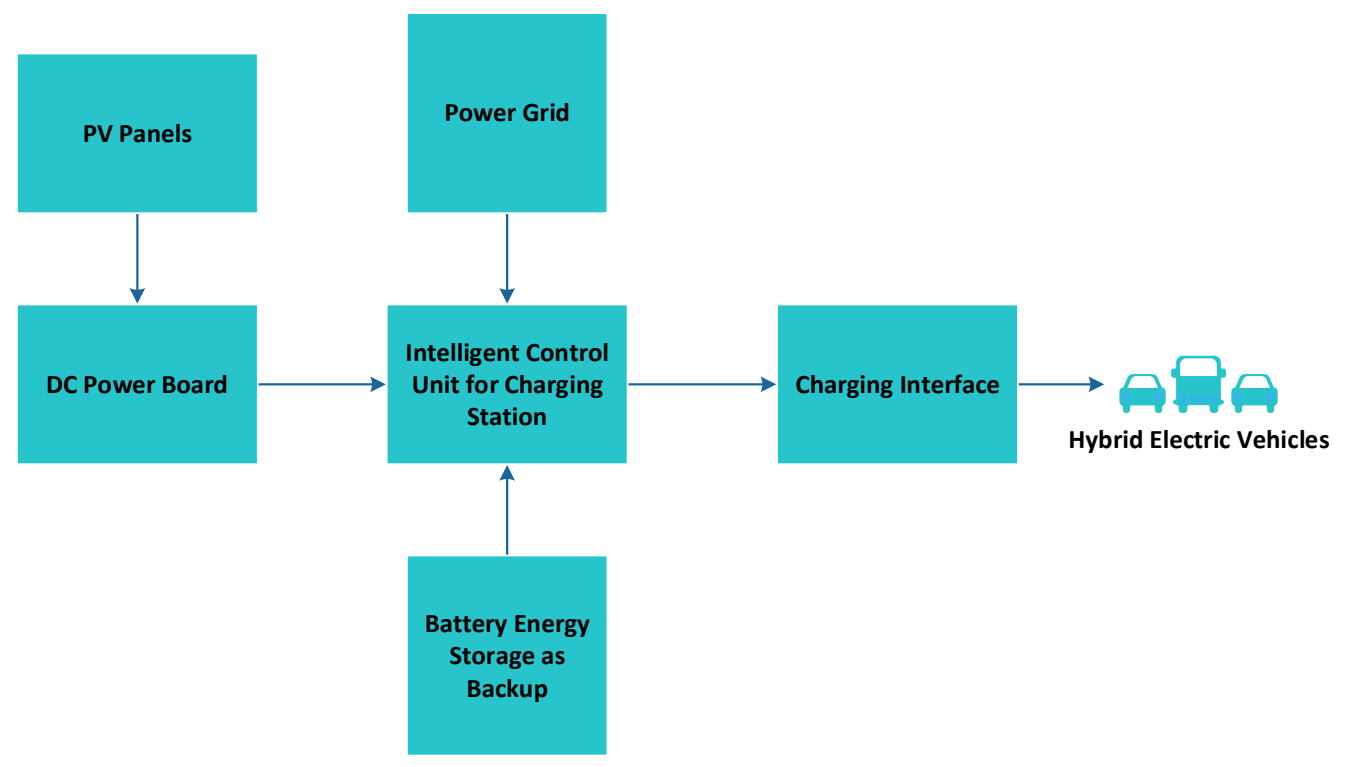

Figure 2. The charging mechanism of solar charging system

\subsection{Constraints}

The objective function, $\mathrm{F}$, is maximized subject to constraints. The first one is that the power demand of an EV cannot exceed the rated power output of the charger.

$$
0 \leq P_{E V i} \leq P_{E V i} \max
$$

To prevent large variations in the charging rate over successive time steps, which is undesirable for battery charging current.

$$
P_{E V_{l}}{ }^{t-1}-\Delta \leq P_{E V_{l}}{ }^{t} \leq P_{E V_{l}}{ }^{t-1}+\Delta
$$


where $t$ is the current time step and $\Delta$ is a defined limit in $k W$. Also, the charging voltage must be maintained within the rated voltage specified for the EV battery, the power output of the charging station is often limited to avoid risks to the charger.

Each EV arrives and departs at a different time, hence, battery capacity and residual battery energy, optimal scheduling of EV charging and discharging is required to determine the priorities of EVs to ensure equality of charging and discharging process, as well as the requirement of adequate energy for every EV before departures.

\section{Results and Discussion}

In this section, we present the developed algorithm for PV-based HEV charging stations. Two different algorithms are developed for HEV and backup battery charging.

\subsection{Control Method of Designed System}

Two-stage charging control techniques are described below considering the followings:

- Per each day, there are two local kWh price peaks, one in the morning and one in the evening. The morning peak is normally lower than the evening peak, i.e. the price of $\mathrm{kWh}$ in the evening is higher.

- Per each day, there is a minimum $\mathrm{kWh}$ price, which is applicable between midnight and sunshine.

- The $\mathrm{kWh}$ prices are normalized in the interval $[0,1]$, so that, the lowest price is provided as 0 and the highest price is provided as 1 .

- The cost of a $\mathrm{kWh}$ from the diesel generator is also normalized to the cost of $\mathrm{kWh}$ from the grid. This value is expected to be greater than one.

- Any surplus power from the EV or the diesel generator is used to charge the batteries

\subsubsection{Algorithm 1: EV charging process}

In this algorithm, the inputs are as follows:

- Grid Energy Status,

- Normalized Grid kWh price,

- Normalized Generator kWh Cost,

- Solar Radiation Level,

- Batteries Level.

Output is the source to be used to charge a HEV.

1. Step 1: GS $\leftarrow$ Grid Energy Status.

$\mathrm{GP} \leftarrow$ Normalized Grid kWh price.

$\mathrm{SR} \leftarrow$ Solar radiation level.

$\mathrm{BL} \leftarrow$ Batteries level.

2. Step 2: Charging using PV

If $\mathrm{BL}>0.5$ and $\mathrm{SR}>0.9$ : If batteries have more than $50 \%$ charge and sufficient $\mathrm{PV}$ radiation exists (in the noon), charging using the PV.

3. Step 3: Charging using PV 
If $\mathrm{BL}>0.9$ and $\mathrm{SR}>0.7$ : If the batteries are almost full, use the EV even at lower level of radiation.

4. Step 4: Charging using Grid If GS and GP < 0.4: If the grid power is available and at low price.

5. Step 5: Charging using Grid

If GS and $\mathrm{BL}<0.6$ and GP < 0.7: If batteries level is less than 0.6 and the cost of the grid $\mathrm{kWh}$ is less than $70 \%$ of peak price, use the grid to reserve batteries for higher grid prices.

6. Step 6: Charging using batteries

If $\mathrm{BL}>0.05$ : If sufficient energy is remaining in the batteries.

7. Step 7: Charge using the diesel generator during the time when there is no sun and the kwh of power grid is higher rate

\subsubsection{Algorithm 2: Battery charging process}

The inputs for algorithm 2 are given below:

- Grid Energy Status,

- Normalized Grid kWh price,

- Solar Radiation Level,

- Batteries Level.

The output is the battery charging decision.

\section{Step 1:}

GS $\leftarrow$ Grid Energy Status.

$\mathrm{GP} \leftarrow$ Normalized Grid $\mathrm{kWh}$ price.

$\mathrm{SR} \leftarrow$ Solar radiation level.

$\mathrm{BL} \leftarrow$ Batteries level

2. Step 2: Battery Charging using PV

If $\mathrm{BL}<1$ and $\mathrm{SR}>0.05$ : //If sunlight exists.

3. Step 3: Battery Charging using grid

If $\mathrm{BL}<0.9$ and $\mathrm{GS}$ and $\mathrm{GP}<0.4$ : //If grid power is available with low price. 


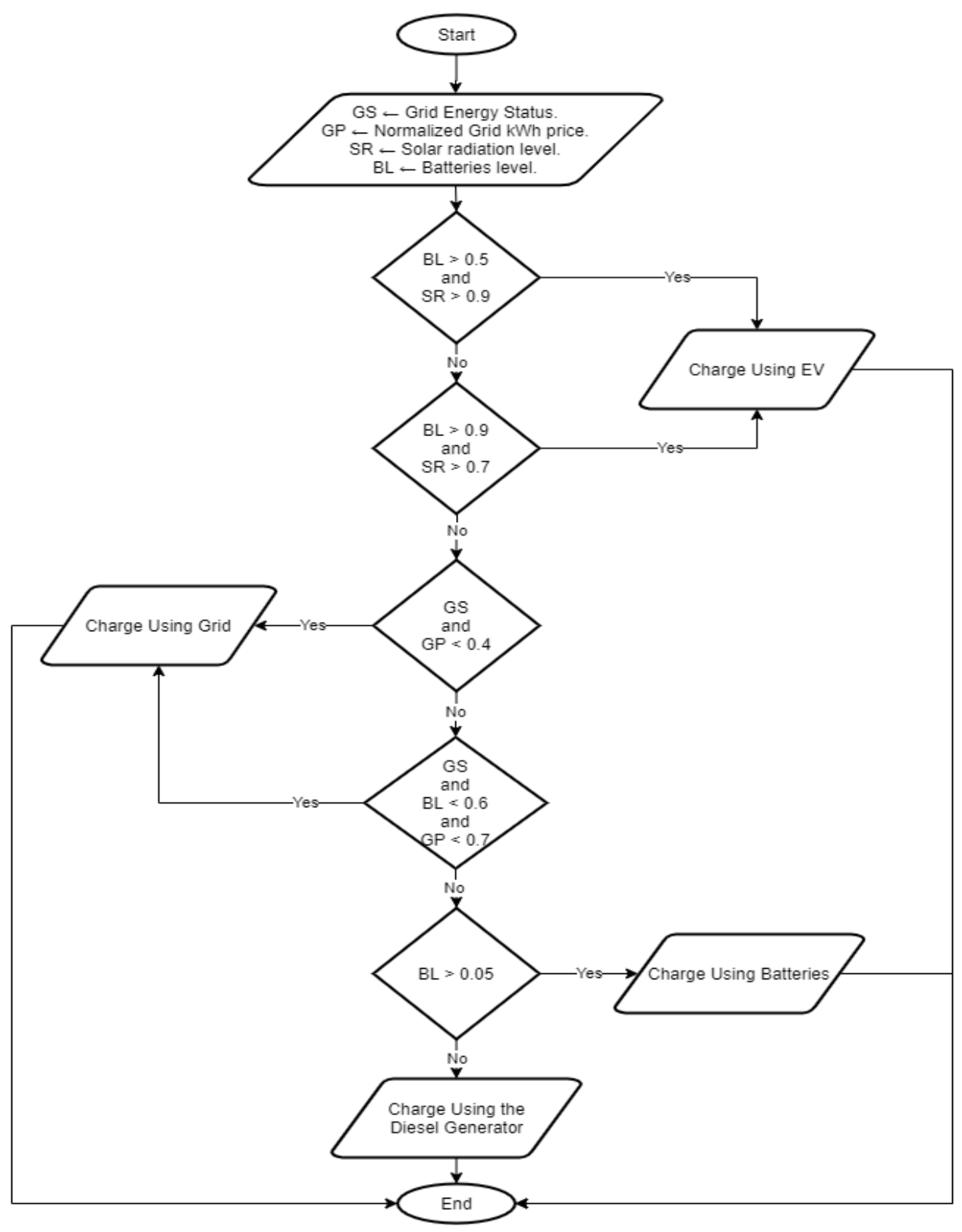

Figure 3. The flowchart of the EV charging algorithm 


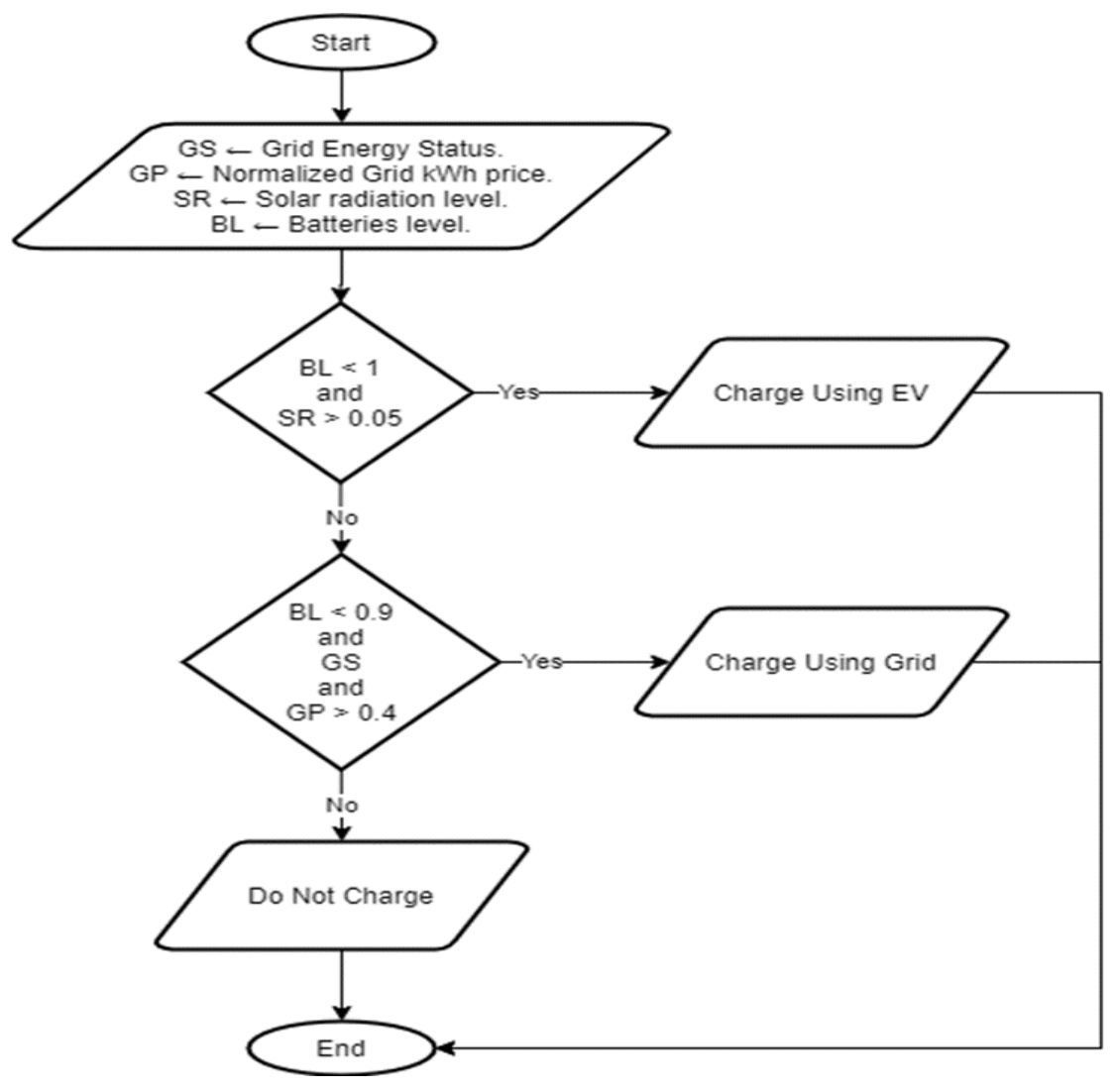

Figure 4. The flowchart of the battery charging algorithm

In order to maximize the benefits of using HEVs, optimized and regulated charging control needs to be provided. In this paper, an analysis of HEV charging from a PV was performed under different charging schemes and two algorithms were applied. An optimal control problem was defined with two different scenarios to handle the problem. The first optimal control problem considered the minimization of $\mathrm{HEV}$ charging cost. In this case, the benefits of V2G were investigated. The process results in significant cost savings compared to the uncontrolled charging. Also, the grid gains from low demand or even available extra power in peak hours. The second control strategy considers the minimization of the power from the grid. The algorithm tends to take advantage of the PV power as much as possible to charge HEVs, which causes minimum impacts on the grid. In this case, the charging costs for HEV is the best option due to the high solar power production and high electricity prices.

\section{Conclusions}

The rapid increase of using HEV batteries connected to the utility grid leads to a heavy burden on the distribution power system and causing load shading. This problem can be solved by the appropriate implementation of solar-based charging stations where photovoltaic panels along with battery storage units are considered. Because of the limited fossil fuel resources in the world and high emissions from conventional girds, this type of stations can be an efficient option for HEV charging when electricity generation cost is higher. Furthermore, solar charging stations are environmentally friendly and has no harmful effect on nature during operation. This system also gives a great opportunity for the appropriate utilization of available solar irradiance in many countries. 


\title{
Acknowledgement
}

The authors acknowledge the support by Karabuk University.

\author{
Nomenclature \\ BESS: Battery Energy Storage System \\ BL : Batteries Level \\ EMS : $\quad$ Energy Management Strategy \\ EV : Electric Vehicle \\ GP : Normalized Grid Price \\ GS : Grid Energy Status \\ HEV : Hybrid Electric Vehicle \\ ICE : Internal Combustion Engine \\ NTRC: The National Transportation Research Center \\ $\mathrm{P}$ : Power \\ SCS : $\quad$ Solar Charging Station \\ SR : Solar Radiation Level \\ V2G : Vehicle-to-Grid
}

\section{References}

[1] Zhang, F., et al., Energy management strategies of connected HEVs and PHEVs: Recent progress and outlook. 2019. 73: p. 235-256.

[2] Onori, S., L. Serrao, and G. Rizzoni, Hybrid electric vehicles: Energy management strategies. 2016: Springer.

[3] Ali, M., S. Mohammad, and M.M. Rahman. Modelling a Solar Charge Station for Electric Vehicle with Storage Backup. in 2019 1st International Conference on Advances in Science, Engineering and Robotics Technology (ICASERT). 2019. IEEE.

[4] Li, L., et al., A novel combinatorial optimization algorithm for energy management strategy of plug-in hybrid electric vehicle. 2017. 354(15): p. 6588-6609.

[5] Tran, D.-D., et al., Thorough state-of-the-art analysis of electric and hybrid vehicle powertrains: Topologies and integrated energy management strategies. 2020. 119: p. 109596.

[6] Wirasingha, S.G. and A.J.I.T.o.v.t. Emadi, Classification and review of control strategies for plug-in hybrid electric vehicles. 2010. 60(1): p. 111-122.

[7] Davis, S.C., S.W. Diegel, and R.G. Boundy, Transportation energy data book. 2009, Oak Ridge National Laboratory.

[8] Ali, T.J.T.D.S., May, Electric Rickshaws run out of steam. 2011. 30.

[9] Jin, C., J. Tang, and P.J.I.T.o.v.t. Ghosh, Optimizing electric vehicle charging: A customer's perspective. 2013. 62(7): p. 2919-2927.

[10] Adika, C.O. and L.J.I.t.o.s.g. Wang, Autonomous appliance scheduling for household energy management. 2013. 5(2): p. 673-682.

[11] Honarmand, M., A. Zakariazadeh, and S.J.E. Jadid, Optimal scheduling of electric vehicles in an intelligent parking lot considering vehicle-to-grid concept and battery condition. 2014. 65: p. 572-579.

[12] Jian, L., et al., A scenario of vehicle-to-grid implementation and its double-layer optimal charging strategy for minimizing load variance within regional smart grids. 2014. 78: p. 508517. 